\title{
Docetaxel/Cyclophosphamide/Trastuzumab Regimen
}

National Cancer Institute

\section{Source}

National Cancer Institute. Docetaxel/Cyclophosphamide/Trastuzumab Regimen. NCI

Thesaurus. Code C138034.

A regimen consisting of docetaxel, cyclophosphamide and trastuzumab, used as a neoadjuvant or adjuvant treatment for HER-2/neu-positive breast cancer. 